\title{
Histopathologic Regression and Survival in RAS Wildtype Metastatic Colorectal Cancer Under First-Line Treatment - Subgroup Analysis of the VOLFI Trial (AIO-KRK-0109)
}

\author{
Stefanie Noepel-Duennebacke ${ }^{1, ~ *}$, Henrik Juette ${ }^{2}$, Celine Lugnier ${ }^{1}$, Dominik Paul Modest ${ }^{3}$, \\ Uwe Martens $^{4}$, Renate Klaassen-Mielke, Volker Heinemann ${ }^{6}$, Thomas Seufferlein ${ }^{7}$, \\ Michael Geissler ${ }^{8}$, Andrea Tannapfel ${ }^{2}$, Anke Reinacher-Schick ${ }^{1}$, Iris Tischoff ${ }^{2}$ \\ ${ }^{1}$ Department of Hematology, Oncology and Palliative Care, St. Josef-Hospital Bochum, Ruhr-University Bochum, Bochum, Germany \\ ${ }^{2}$ Institute of Pathology, Ruhr-University Bochum, Bochum, Germany \\ ${ }^{3}$ Department of Hematology, Oncology, and Tumorimmunology (CVK/CCM), University Medicine Charite, Berlin, Germany \\ ${ }^{4}$ Department of Medicine, SLK-Kliniken Heilbronn, Heilbronn, Germany \\ ${ }^{5}$ Department of Medical Informatics, Biometry and Epidemiology, Ruhr University Bochum, Bochum, Germany \\ ${ }^{6}$ German Cancer Consortium (DKTK), German Cancer Research Centre (DKFZ), Heidelberg, Germany \\ ${ }^{7}$ Department of Medicine I, Hospital of the University, Ulm, Germany \\ ${ }^{8}$ Department of Hematology/Oncology, Clinic of Esslingen, Esslingen Germany
}

Email address:

stefanie.noepel-duennebacke@rub.de (S. Noepel-Duennebacke)

${ }^{*}$ Corresponding author

To cite this article:

Stefanie Noepel-Duennebacke, Henrik Juette, Celine Lugnier, Dominik Paul Modest, Uwe Martens, Renate Klaassen-Mielke, Volker Heinemann, Thomas Seufferlein, Michael Geissler, Andrea Tannapfel, Anke Reinacher-Schick, Iris Tischoff. Histopathologic Regression and Survival in RAS Wildtype Metastatic Colorectal Cancer Under First-Line Treatment - Subgroup Analysis of the VOLFI Trial (AIO-KRK-0109). International Journal of Clinical Oncology and Cancer Research. Vol. 6, No. 3, 2021, pp. 130-135.

doi: 10.11648/j.ijcocr.20210603.15

Received: June 24, 2021; Accepted: July 12, 2021; Published: July 27, 2021

\begin{abstract}
Aim: The VOLFI trial demonstrated an improved objective response rate through the addition of panitumumab to FOLFOXIRI in untreated all-RAS-wildtype mCRCs compared to FOLFOXIRI alone. In this subgroup analysis, we focused on histopathological response as a predictive marker for PFS. Additionally, we analyzed chemotherapy induced steatosis hepatitis (CASH) in both treatment arms. Methods: Histopathological response, CASH, sinusoidal obstructive syndrome, ballooning, steatosis, cholestasis, fibrosis and inflammation were determined in 14 resected liver metastasis. PFS was estimated using Kaplan-Meier method, the logrank test was used for the statistical comparison. The trial is registered with Clinical Trials. gov, NCT01328171. Results: Tissue of 14/18 resected pts. was evaluable. Median age was 57.5 yrs. (32-67), 7 male and 7 females. All primary tumors were located in the left colon. Molecular analysis detected one BRAF V600E mutation and one MSI-H tumor. Median treatment duration until resection were 7 cycles $(3-12)$ panitumumab/mFOLFOXIRI and 9.5 cycles $(7$ - 11) FOLFOXIRI. 7 pts. achieved very good histopathological response corresponding to $\leq 20 \%$ vital tumor cells (panitumumab/ $\mathrm{mFOLFOXIRI} \mathrm{vs.}$ FOLFOXIRI 2/5) and 7 pts. showed vital tumor cells $>20 \%$ (panitumumab/mFOLFOXIRI vs. FOLFOXIRI 2/5). A very good histopathological response (residual tumor cells in proportion to the total tumor area $\leq 20 \%$ ) showed a trend to an improved PFS in comparison to $>20 \%$ (median PFS 12.40; 95\% CI 6.43-51.22 vs. PFS 9.88; 95\% CI 6.17-15.26 months). The severity of CASH was not increased by the addition of panitumumab or longer duration of chemotherapy. Discussion: In this analysis histopathological response seems to correlate with a better PFS after secondary metastasis resection. By analysis of liver toxicity, no relevant difference of CASH were detectable regarding panitumumab/mFOLFOXIRI vs. FOLFOXIRI or the duration of chemotherapy.
\end{abstract}

Keywords: Metastatic Colorectal Cancer, Regression Rate, Panitumumab, RAS, CASH 


\section{Introduction}

Early tumor shrinkage (ETS) is an often intended goal in the first line therapy of metastatic colorectal cancer (mCRC). It predicts progression free survival (PFS) and overall survival (OS) and is therefore a potential positive prognostic marker for first line regimens in mCRC [1, 2]. Furthermore, ETS can lead to secondary resections (SR) of metastases. Within this treatment concept a chance of cure may be offered even in a metastatic setting. To establish ETS and or SR and thereby increase survival an intensive chemotherapy regimen comprising of fluorouracil/folinic acid, irinotecan and oxaliplatin (FOLFOXIRI) is recommended if feasible [3]. If SR is not possible, ETS can decrease a high tumor load, reduce tumor related symptoms and improve the quality of life. FOLFOXIRI is an intense and active upfront treatment regimen for mCRCs [3]. Combined with an anti-VEGF-antibody (Bevacizumab) it prolongs OS and leads to a higher response rate (RR) with tolerable toxicity in comparison to bevacizumab/FOLFIRI [1]. In the VOLFI trial we found a benefit regarding ORR when adding the anti-EGFR-antibody panitumumab to the chemotherapy backbone FOLFOXIRI. The VOLFI trial, was the first to investigate the impact of adding an anti EGFR-antibody (Panitumumab, arm A) to FOLFOXIRI (Arm B) in the first line setting in $\mathrm{mCRC}$. This regimen lead to a higher objective response rate (ORR) in the experimental arm of $87.3 \%$ vs. $60.6 \%$ (OR 4.47; $95 \%$ CI 1.614-12.376; $\mathrm{p}=0.0041$ ), and to higher rate of SR of metastases in the panitumumab containing arm A. OS (ITT population) showed a strong trend in favor of arm A with a median OS of 35.7 vs. 29.8 months in arm B (HR: 0.67; 95\% CI 0.41-1.11, $\mathrm{p}=0.12$ ) [4]. The VOLFI trial demonstrated that intensified therapy regimens in suitable pts. can lead to a higher rate of cure with SR. However, higher intensity and dose modification of cytotoxic agents can lead to adverse events. Irinotecan- and Oxaliplatin-containing chemotherapy can induce severe side effects like chemotherapy induced steatosis hepatis (CASH) and sinusoidal obstruction syndrome (SOS), [5]. Their incidences vary between $20-50 \%[6,7]$. Both can lead to reduced or even crucial liver function impairment and may increase the perioperative morbidity and mortality $[8,9]$. CASH induced by irinotecan appears in 1 of 5 pts. and its histopathological appearance is a severe form of non-alcoholic steatohepatitis (NASH) with inflammation and possible fibrosis / cirrhosis. Imaging techniques cannot distinguish between simple NASH from CASH; therefore, biopsy and histopathological analysis is mandatory [10]. The histopathological characteristics of SOS are hepatic atrophy, nodular regenerate hyperplasia, hepatic sinusoidal dilation and perisinusoidal fibrosis. Its manifestation is scored from 0 (absent) to 3 (severe; 11). Oxaliplatin can lead to SOS in at least $50 \%$ of pts. receiving chemotherapy before resection and is a key risk factor for postoperative morbidity [7. 9, 12, 13]. Meanwhile, the association of chemotherapy and liver damage is apparent, while the liver toxicity of anti-EGFR agents like panitumumab is not known [14].

In this subgroup analysis of the VOLFI trial (AIO-KRK-0109), we focused on histopathological response as a predictive marker for PFS. Additionally, we analyzed hepatologic toxicity in both treatment arms.
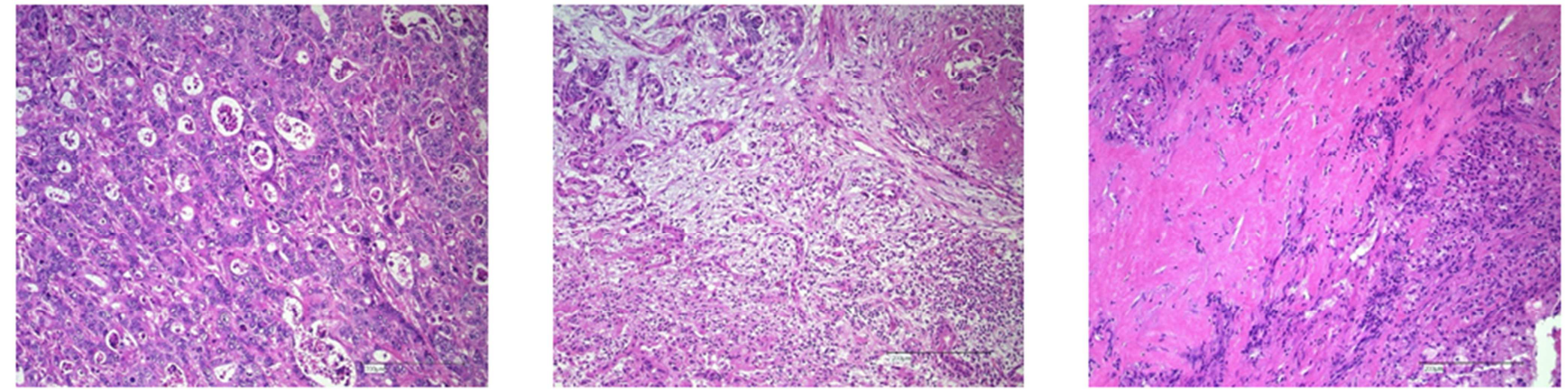

Figure 1. Liver metastasis with no (A), strong (B) and complete (C) regression analougous to regression grading of Rubbia-Brandt.
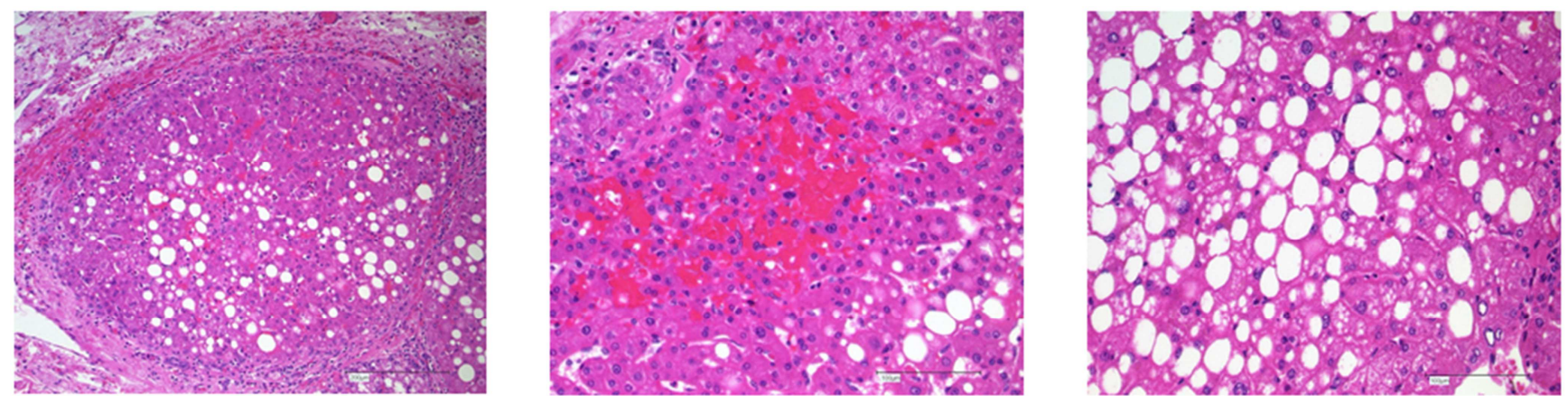

Figure 2. H\&E (histology) staining of chemotherapy induced toxicity: CASH (A), CASH with SOS (B) and steatosis hepatis and CASH (C), CASH: chemotherapy induced steatosis hepatitis, SOS: sinusoidal obstructive syndrome. 


\section{Methods}

The phase II controlled, open label multi-center trial VOLFI (AIO-KRK-0109, NCT01328171) randomized prospectively 2:1 untreated all-RAS-WT mCRC pts. comparing panitumumab/mFOLFOXIRI (arm A) versus FOLFOXIRI (Arm B). Prospective strata were two cohorts: 1 irresectable mCRC, which was unnoticed in this subgroup analysis and cohort 2: chance to SR of metastatic lesions in curative intent. Primary endpoint of the trial was ORR, secondary endpoint was resection rate (cohort 2), disease control rate (DCR), PFS and OS. Treatment was administered until progression (PD), resectability or to a maximum of 12 cycles. If SR was achieved, surgery was performed and the protocol treatment was proceeded up to 12 cycles [4]. Out of cohort $218 / 31$ pts. achieved SR of metastatic liver lesions. 14 tissue samples from liver metastases resection were available and analyzed within this substudy. The following histopathological markers were examined: residual tumor cells (\%), response rate analogues to Blazer [15] and Rubbia-Brandt (11; Figure 1). For chemotherapy induced liver toxicity following histopathological parameters were analyzed: SOS, steatosis hepatis, ballooning, cholestasis, fibrosis and inflammation were determined and classified analogous to previously published scoring systems $[11,16]$. CASH was assessed using the NAS score comprising the rate of steatosis, inflammation and ballooning [17], (Figure 2). We defined a cut-off for very good histopathological response at $20 \%$ of residual tumor cells in proportion to the total tumor area. PFS was estimated using Kaplan-Meier method, the logrank test was used for the statistical comparison. Statistical analyses were done using SAS software, version 9.4 .

\section{Results}

The results from the VOLFI trial were published previously. Adding panitumumab to a first line treatment with FOLFOXIRI in all-RAS WT mCRC pts. increased the ORR from $60.6 \%$ to $87.3 \%$. This led to a higher rate of SR of liver metastases in the panitumumab group of $33.3 \%$ vs. $12.1 \%$ and showed a trend to a longer median OS (HR 0.413; 95\%-CI 0.15-1.12, $\mathrm{p}=0.07$ ) in the group of pts. with intended SR [4].

This subgroup analysis regarding histopathological regression comprised pts. from cohort 2 (chance to SR of metastatic lesions in curative intent) undergoing secondary liver metastases resection with available tumor tissue. Overall 18 pts. achieved secondary resection, 14 tissue probes out of these 18 pts. were available and analyzed. Concerning both treatment arms 10 pts. received panitumumab/mFOLFOXIRI (arm A) and 4 FOLFOXIRI (arm B). The median age was 57.5 yrs. (3267), comprising 7 male and 7 females. All primary tumors were located in the left colon. Molecular analysis detected one BRAF (V600E) mutation and one MSI-H tumor, both we randomized in $\operatorname{arm} \mathrm{A}$. Median treatment duration until resection in this cohort 2 was 7 cycles $(3-12)$ for panitumumab/mFOLFOXIRI vs. 9.5 cycles $(7-11)$ for FOLFOXIRI alone. 7 pts. achieved a very good histopathological response corresponding vital tumor cells $\leq 20 \%$ (panitumumab/mFOLFOXIRI: 5 vs. FOLFOXIRI: 2) and 7 pts. showed vital tumor cells $>20 \%$ (panitumumab/mFOLFOXIRI 5 vs. FOLFOXIRI: 2; Table 1). In our analysis pts. with very good histopathological response (residual tumor cells in proportion to the total tumor area $\leq 20 \%$ ) showed a trend to an improved PFS in comparison to $>20 \%$ residual tumor cells. The median PFS was 12.4 months $(95 \%$ confidence interval (CI) 6.4 - 51.2) vs. 9.9 months (95\% CI 6.2 15.3 months, $p=0.26$, Figure 3 ). All but 1 resected pts. showed a partial remission $(\mathrm{pR})$ measured via CT imaging using Response Evaluation Criteria in Solid Tumors; RECIST criteria 1.1. Histopathological regression rate using the Blazer scoring system detected 8/10 major responses in arm $\mathrm{A}$ and 3/4 in arm B. The incidence of steatosis hepatis in arm A was $6 / 10$ vs. $2 / 4$ in arm B. 5/10 pts. in arm A had a cholestasis whereas $1 / 4$ pt. in arm B. SOS was detected in $5 / 10$ pts. among panitumumab/mFOLFOXIRI and in $2 / 4$ pt. under FOLFOXIRI. In relation to liver toxicity CASH did not differ between both treatment arms nor histological response rates (Rubbia Brandt and Blazer, Table 1). The severity of CASH did not increase by the addition of panitumumab or longer duration of chemotherapy. Of note only the subgroups comprised a small number of pts.

Table 1. Baseline characteristics, treatment, histopathological response and liver toxicity.

\begin{tabular}{|c|c|c|c|c|c|c|c|c|c|c|c|c|c|}
\hline Number & Age & Sex & ECOG & Arm & Localization & $\begin{array}{l}\text { applied } \\
\text { chemotherapy } \\
\text { cycles }\end{array}$ & $\begin{array}{l}\text { RAS } \\
\text { WT }\end{array}$ & BRAF-WT & BRAF-MT & MSI-H & MSS & $\begin{array}{l}\text { Response } \\
\text { (RECIST) }\end{array}$ & $\begin{array}{l}\text { vital } \\
\text { tumor } \\
(\%)\end{array}$ \\
\hline 1 & 51 & M & 0 & B & $\mathrm{L}$ & 9 & $\mathrm{X}$ & $\mathrm{X}$ & & & $\mathrm{X}$ & SD & $75 \%$ \\
\hline 2 & 61 & M & 0 & A & $\mathrm{L}$ & 4 & $\mathrm{X}$ & $\mathrm{X}$ & & & $\mathrm{X}$ & $\mathrm{pR}$ & $40 \%$ \\
\hline 3 & 33 & W & 0 & A & $\mathrm{L}$ & 7 & $\mathrm{X}$ & & $\mathrm{X}$ & & $\mathrm{X}$ & $\mathrm{pR}$ & $<10 \%$ \\
\hline 4 & 60 & M & 0 & A & $\mathrm{L}$ & 12 & $\mathrm{X}$ & $\mathrm{X}$ & & & $\mathrm{X}$ & $\mathrm{pR}$ & $20 \%$ \\
\hline 5 & 63 & M & 1 & B & $\mathrm{L}$ & 10 & $X$ & $\mathrm{X}$ & & & $\mathrm{X}$ & $\mathrm{pR}$ & $5 \%$ \\
\hline 6 & 55 & W & 1 & B & $\mathrm{L}$ & 11 & $X$ & $\mathrm{X}$ & & & $\mathrm{X}$ & $\mathrm{pR}$ & $30 \%$ \\
\hline 7 & 52 & M & 0 & A & $\mathrm{L}$ & 8 & $X$ & $\mathrm{X}$ & & & $\mathrm{X}$ & $\mathrm{pR}$ & $75 \%$ \\
\hline 8 & 60 & W & 0 & A & $\mathrm{L}$ & 7 & $\mathrm{X}$ & $\mathrm{X}$ & & & $\mathrm{X}$ & $\mathrm{pR}$ & $<5 \%$ \\
\hline 9 & 61 & W & 0 & B & $\mathrm{L}$ & 7 & $\mathrm{X}$ & $\mathrm{X}$ & & & $\mathrm{X}$ & $\mathrm{pR}$ & $<5 \%$ \\
\hline 10 & 47 & W & 0 & A & $\mathrm{L}$ & 4 & $\mathrm{X}$ & $\mathrm{X}$ & & $\mathrm{X}$ & & $\mathrm{pR}$ & $95 \%$ \\
\hline 11 & 41 & W & 0 & A & L & 8 & $\mathrm{X}$ & $\mathrm{X}$ & & & $\mathrm{X}$ & $\mathrm{pR}$ & $30 \%$ \\
\hline 12 & 36 & M & 0 & A & $\mathrm{L}$ & 3 & $X$ & $\mathrm{X}$ & & & $\mathrm{X}$ & $\mathrm{pR}$ & $40 \%$ \\
\hline 13 & 61 & M & 0 & A & $\mathrm{L}$ & 9 & $\mathrm{X}$ & $\mathrm{X}$ & & & $\mathrm{X}$ & $\mathrm{pR}$ & $<5 \%$ \\
\hline 14 & 67 & W & 0 & A & $\mathrm{L}$ & 7 & $\mathrm{X}$ & $\mathrm{X}$ & & & $\mathrm{X}$ & $\mathrm{pR}$ & $15 \%$ \\
\hline
\end{tabular}


Table 1. Continued.

\begin{tabular}{|c|c|c|c|c|c|c|c|c|c|c|}
\hline Number & $\begin{array}{l}\text { Response (Blazer } \\
\text { et al.) }\end{array}$ & $\begin{array}{l}\text { Regression } \\
\text { (Rubbia-Brandt } \\
\text { et al.) }\end{array}$ & Steatosis & Inflammation & Ballooning & Cholestasis & SOS & Fibrosis & CASH & $\begin{array}{l}\text { PFS } \\
\text { (days) }\end{array}$ \\
\hline 1 & Minor-Response & 4 & 3 & 2 & 2 & 0 & 0 & 3 & 7 & 235 \\
\hline 2 & Major-Response & 3 & 1 & 2 & 1 & 0 & 0 & 2 & 4 & 188 \\
\hline 3 & Major-Response & 3 & 0 & 3 & 0 & 1 & 0 & 2 & 4 & 196 \\
\hline 4 & Major-Response & 3 & 1 & 2 & 2 & 0 & 0 & 2 & 5 & 378 \\
\hline 5 & Major-Response & 3 & 1 & 2 & 1 & 0 & 0 & 2 & 4 & 1561 \\
\hline 6 & Major-Response & 3 & 0 & 2 & 0 & 1 & 2 & $1 \mathrm{C}$ & 5 & 300 \\
\hline 7 & Minor-Response & 4 & 1 & 2 & 1 & 1 & 2 & 2 & 7 & 301 \\
\hline 8 & Major-Response & 2 & 0 & 2 & 0 & 2 & 1 & 2 & 5 & 1336 \\
\hline 9 & Major-Response & 2 & 0 & 2 & 0 & 2 & 1 & $1 \mathrm{C}$ & 5 & 266 \\
\hline 10 & Minor-Response & 4 & 3 & 2 & 2 & 0 & 0 & $1 \mathrm{C}$ & 7 & 465 \\
\hline 11 & Major-Response & 3 & 0 & 2 & 0 & 1 & 1 & 1B & 4 & 328 \\
\hline 12 & Major-Response & 3 & 0 & 2 & 0 & 2 & 2 & 4 & 6 & 763 \\
\hline 13 & Major-Response & 3 & 1 & 2 & 0 & 2 & 1 & 2 & 6 & 391 \\
\hline 14 & Major-Response & 3 & 3 & 3 & 2 & 1 & 0 & 4 & 9 & 373 \\
\hline
\end{tabular}

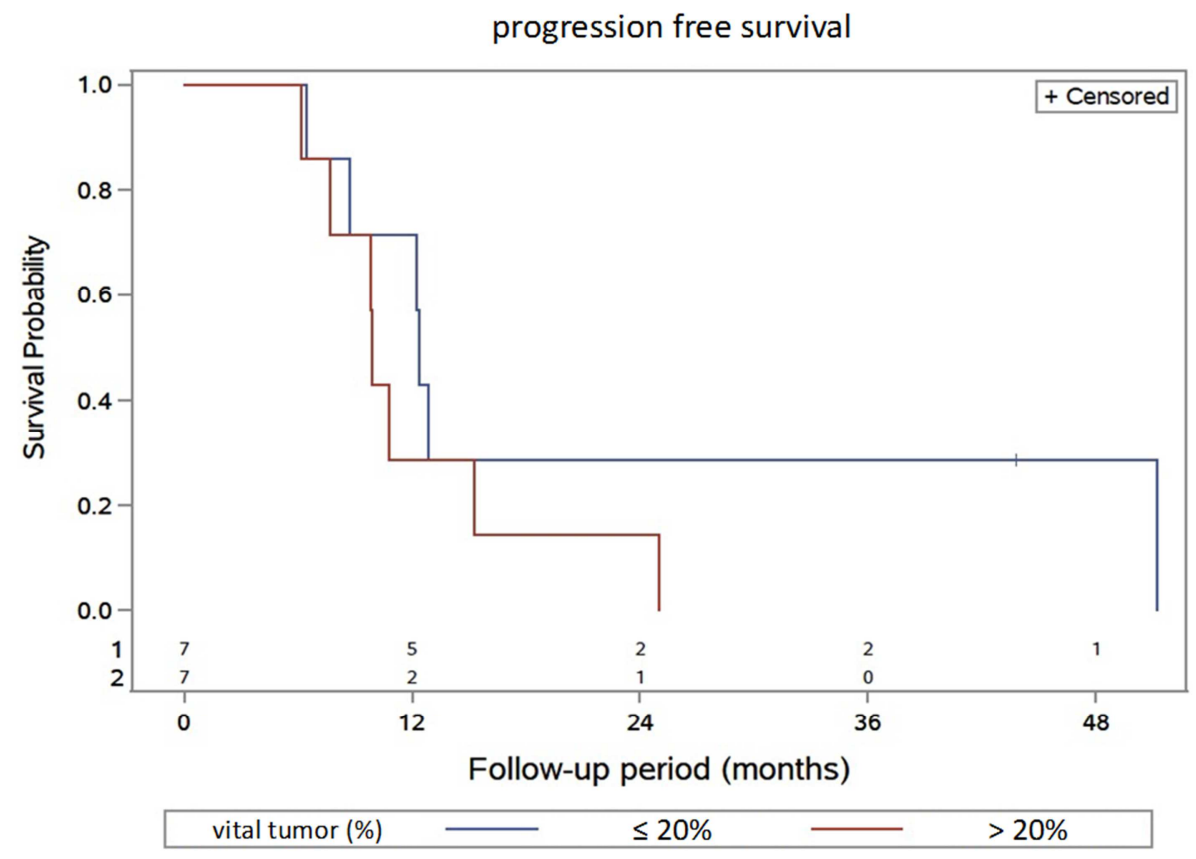

Figure 3. Progression free survival in relation to residual vital tumor cells in resected liver metastasis.

\section{Discussion}

The VOLFI trial (NCT01328171) gave evidence that the addition of panitumumab to mFOLFOXIRI in all-RAS-WT mCRC significantly improved the ORR $(87.3 \%$ vs $60.6 \%$, odds ratio 4.469 (95\% CI 1.61-12.38, $\mathrm{p}=0.004)$ and the rate of secondary resection of liver metastases $(33.4 \%$ vs. $12.1 \%$, $\mathrm{p}=0.02$ ). Furthermore, a trend towards improved OS in the panitumumab group was obvious (HR for death 0.67 ; $(95 \% \mathrm{CI}$ $0.41-1.11, p=0.12 ; 4)$. In this subgroup analysis the histopathological response with $\leq 20 \%$ residual tumor cells showed a trend for an improved PFS and anti-EGFR treatment did not aggravate liver toxicity. To establish curative treatment strategies in mCRCs an intensified first line treatment if feasible is recommended [18]. To enable SR of liver metastasis highly effective cytotoxic regimen (FOLFOXIRI) and antibodies such as anti-EGFR are clearly indicated if suitable $[1,3]$. But their side effects explicitly liver toxicity needs to be noticed prior to surgery. CASH may be crucial for further liver function and therefore increase perioperative mortality and morbidity. Irinotecan mainly causes steatohepatitis with inflammation, fibrosis and cirrhosis and oxaliplatin effects SOS. 5-Fluorouracil (5FU) can also affect steatohepatitis but its incidence is much lower compared to irinotecan $[5,17]$. Up to date, the pathogenesis of 5-FU and irinotecan induced adversities are not fully understood, but hypotheses suggest a damage in mitochondria membranes which lead to disrupting of the oxidation of fatty acids and causing fat accumulating inside the hepatocytes [5]. If steatosis hepatis exceeds $30 \%$ the risk of death after major resection increases nearly threefold [19]. SOS is associated with following histopathological changes in hepatic cells: hepatic sinusoidal dilation, hepatocyte atrophy, perisinusoidal fibrosis, and nodular regenerative hyperplasia. These damages can be graded using a scoring system by Rubbia-Brandt et al. 
$[11,16]$. According to CASH the pathogenesis of SOS remains unclear. Risk factors for the development of chemotherapy induced liver toxicity may be the duration of therapy [20], the time interval to surgery [9] and preexisting liver damage. Liver function needs to be tested before SR. If a biopsy is practical, the tumor regression using Rubbia-Brandt and residual tumor cell percentage should be performed. Within these analyses the RR is objectifiable in comparison to imaging techniques and was recommended previously [21].

Imaging procedures cannot distinguish between simple NASH from CASH, but MRI imaging may detect specific SOS changes. Therefore, a biopsy and histopathological analysis are mandatory and should be performed prior to surgery $[10,22,23]$. In our data there was no evidence of panitumumab induced liver toxicity which is conform with previously published data [14]. Anti-EGFR treatment is highly effective in all-RAS-WT mCRC pts. and should be used if indicated and feasible to establish ETS and aim for SR in curative intent. Nevertheless, up to date CT and MRI is the gold standard to define remission under anti-cancer treatment but considering SR in curative intent more attention must be payed with respect to $\mathrm{CASH}$.

In this small subgroup analysis of secondary resected liver metastasis among all-RAS-WT mCRC pts. undergoing first line treatment with either FOLFOXIRI or panitumumab/mFOLFOXIRI we detected a trend to an improved PFS among pts. with $\leq 20 \%$ vital tumor cells. Concerning CASH and liver toxicity, comparing both treatment arms, this subgroup analysis did not demonstrate essential differences. Because of the small sample size, the statistical significance could not be proven and thereby these findings need to be validated in a greater cohort.

\section{Conclusion}

Concerning chemotherapy induced liver toxicity histopathological analysis are the most reliable to detect sufficiently specific side effects like CASH and SOS. Additionally, the histopathological response may officiate as marker for further treatment strategies after liver metastasis resection because of its putative prognostic value.

\section{References}

[1] Cremolini C, Loupakis F, Antoniotti C, Lonardi S. Early tumor shrinkage and depth of response predict long-term outcome in metastatic colorectal cancer patients treated with first-line chemotherapy plus bevacizumab: results from phase III TRIBE trial by the Gruppo Oncologico del Nord Ovest. Ann Oncol. 2015 Jun; 26 (6): 1188-94.

[2] Stintzing S, Modest DP, Rossius L et al. FOLFIRI plus cetuximab versus FOLFIRI plus bevacizumab for metastatic colorectal cancer (FIRE-3): a post-hoc analysis of tumour dynamics in the final RAS wild-type subgroup of this randomised open-label phase 3 trial. Lancet Oncol. 2016 Oct; 17 (10): 1426-1434. doi: 10.1016/S1470-2045(16)30269-8. Epub 2016 Aug 27. Erratum in: Lancet Oncol. 2016 Oct; 17 (10): e420. Erratum in: Lancet Oncol. 2016 Nov; 17 (11): e479.

\section{PMID: 27575024.}

[3] Moretto R, Rossini D, Zucchelli G, et al. Oligometastatic colorectal cancer: prognosis, role of locoregional treatments and impact of first-line chemotherapy-a pooled analysis of TRIBE and TRIBE2 studies by Gruppo Oncologico del Nord Ovest. Eur J Cancer. 2020 Nov; 139: 81-89. doi: 10.1016/j.ejca.2020.08.009. Epub 2020 Sep 23. PMID: 32979645 .

[4] Modest DP, Martens UM, Riera-Knorrenschild J, et al. FOLFOXIRI Plus Panitumumab As First-Line Treatment of RAS Wild-Type Metastatic Colorectal Cancer: The Randomized, Open-Label, Phase II VOLFI Study (AIO KRK0109). J Clin Oncol. 2019; 37 (35): 3401-3411. doi: 10.1200/JCO.19.01340.

[5] Duwe G, Knitter S, Pesthy S, et al. Hepatotoxicity following systemic therapy for colorectal liver metastases and the impact of chemotherapy-associated liver injury on outcomes after curative liver resection. Eur J Surg Oncol. 2017; 43 (9): 1668 1681. doi: 10.1016/j.ejso.2017.05.008.

[6] Morris-Stiff, G, Tan, Y-M, Vauthey JN, et al. Hepatic complications following preoperative chemotherapy with oxaliplatin or irinotecan for hepatic colorectal metastases. Eur J Surg Oncol. 2008 Jun; 34 (6): 609-14. Epub 2007 Aug 30.

[7] Viganò L, Capussotti L, De Rosa G, De Saussure WO, Mentha G, Rubbia-Brandt L. Liver resection for colorectal metastases after chemotherapy: impact of chemotherapy-related liver injuries, pathological tumor response, and micrometastases on long-term survival. Ann Surg 2013; 258: 731-40; discussion

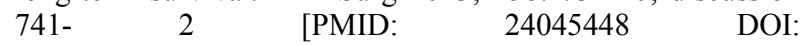
10.1097/SLA.0b013e3182a6183e].

[8] Pandey P, Pandey A, Dillhoff M, et al., Assessing the Non-tumorous Liver: Implications for Patient Management and Surgical Therapy. J Gastrointest Surg. 2018 Feb; 22 (2): 344-360. doi: 10.1007/s11605-017-3562-3. Epub 2017 Sep 18.

[9] Zhao J, van Mierlo KMC, Gómez-Ramírez J, et al. Systematic review of the influence of chemotherapy-associated liver injury on outcome after partial hepatectomy for colorectal liver metastases. Br J Surg. 2017; 104 (8): 990-1002. doi: 10.1002/bjs.10572.

[10] Pilgrim CH, Thomson BN, Banting S, Phillips WA, Michael M The developing clinical problem of chemotherapy-induced hepatic injury. ANZ J Surg 2012; 82 (1e2): 23-9.

[11] Rubbia-Brandt L, Audard V, Sartoretti P, et al. Severe hepatic sinusoidal obstruction associated with oxaliplatin-based chemotherapy in patients with metastatic colorectal cancer. Ann Oncol 2004; 15 (3): 460-6.

[12] Grigorian A and O'Brien CB. Hepatotoxicity Secondary to Chemotherapy. J Clin Transl Hepatol. 2014 Jun; 2 (2): 95-102. Published online 2014 Jun 15. doi: 10.14218/JCTH.2014.00011.

[13] Moreno Prats M, Sasatomi E, Stevenson HL. Colorectal Liver Metastases: A Pathologist's Guide to Creating an Informative Report and Improving Patient Care. Arch Pathol Lab Med. 2019 Feb; 143 (2): 251-257. doi: 10.5858/arpa.2017-0505-RA. Epub 2018 May 23. PMID: 29790787.

[14] Keating GM. Panitumumab: a review of its use in metastatic colorectal cancer. Drugs. 2010 May 28; 70 (8): 1059-78. doi: 10.2165/11205090-000000000-00000. 
[15] Blazer III DG, Kishi Y, Dipen MM et al., Pathologic Response to Preoperative Chemotherapy: A New Outcome End Point After Resection of Hepatic Colorectal Metastases. J Clin Oncol 2008, 25: 5344-5351; DOI: 10.1200/JCO.2008.17.5299.

[16] Van der Bol JM, Mathijssen RHJ, Creemers GJM, Planting AST, Loos WJ, Wiemer EAC, et al. A CYP3A4 phenotype-based dosing algorithm for individ-ualized treatment of irinotecan. Clin Cancer Res Off J Am Assoc Cancer Res2010; 16 (2): 736-42.

[17] Meunier L and Larrey D. Chemotherapy-associated steatohepatitis. Ann Hepatol. 2020 Jan 30. pii: S1665-2681(20)30004-1. doi: 10.1016/j.aohep.2019.11.012.

[18] Schmiegel W, Buchberger B, Follmann M et al. (2017) Z Gastroenterol 55: 1344-1498.

[19] Robinson SM, Wilson $\mathrm{CH}$, Burt $\mathrm{AD}$, et al. Chemotherapy-associated liver injury in patients with colorectal liver metastases: a systematic review and meta-analysis. Ann Surg Oncol 2012; 19 (13): 4287-99.

[20] Kishi Y, Zorzi D, Contreras CM, Maru DM, Kopetz S, Ribero
D, Motta M, Ravarino N, Risio M, Curley SA, Abdalla EK, Capussotti L, Vauthey JN. Extended preoperative chemotherapy does not improve pathologic response and increases postoperative liver insufficiency after hepatic resection for colorectal liver metastases. Ann Surg Oncol 2010; 17: 2870-2876 [PMID: 20567921 DOI: 10.1245/s10434-010-1166-1].

[21] Rubbia-Brandt L, Giostra E, Brezault C et al., Importance of histological tumor response assessment in predicting the outcome in patients with colorectal liver metastases treated with neo-adjuvant chemotherapy followed by liver surgery. Ann Oncol. 2007 Feb; 18 (2): 299-304. Epub 2006 Oct 23.

[22] Zhang Y, Jiang HY, Wei Y, Song B. Sinusoidal obstruction syndrome: A systematic review of etiologies, clinical symptoms, and magnetic resonance imaging features. World $\mathrm{J}$ Clin Cases. 2019 Sep 26; 7 (18): 2746-2759. doi: 10.12998/wjcc.v7.i18.2746.

[23] Zhang Y, Yan Y, Song B. Noninvasive imaging diagnosis of sinusoidal obstruction syndrome: a pictorial review. Insights $\begin{array}{lllll}\text { Imaging. } 2019 \text { Nov 20; } 10 & \text { (1): } 110 . \quad \text { doi: }\end{array}$ 10.1186/s13244-019-0791-x. 